\title{
Diferencias en el metabolismo lipídico, niveles de homocisteína, folato y vitamina B12 entre gestaciones gemelares y únicas
}

\author{
Marcos Cuerva G. ${ }^{1}$, María del Mar Gil M. ${ }^{1}$, Sara lacoponi ${ }^{1}$, María De La Calle F. ${ }^{1}$, \\ Antonio González G. ${ }^{1}$ \\ ${ }^{1}$ Departamento de Ginecología y Obstetricia, Hospital Universitario La Paz. Madrid, España.
}

\section{RESUMEN}

Antecedentes: Se están usando diferentes tipos de suplementación nutricional durante la gestación, sin embargo, existen escasez de estudios que se centren en los resultados metabólicos de los mismos. Objetivos: Comprobar si mediante la suplementación se logra el control del impacto de los múltiples cambios metabólicos que conlleva una gestación múltiple, usando análisis comunes. Método: Comparamos tres diferentes grupos de pacientes entre la semana gestacional 25 y 27 . Un grupo de 46 gestaciones únicas, un grupo de 38 gestaciones gemelares espontáneas y otro grupo de 32 gestaciones gemelares tras técnicas de reproducción asistida. Comparamos niveles de colesterol LDL, HDL, triglicéridos, folato sérico, vitamina B12, homocisteína, hemoglobina, creatinina y plaquetas. Resultados: Los niveles de triglicéridos fueron más altos en ambos grupos de gemelares $(p<0,001)$ y los niveles de colesterol fueron similares en los tres grupos. Las concentraciones de folato sérico fueron mayores en las gestaciones gemelares $(p<0,001)$. El resto de parámetros estudiados fueron similares en los tres grupos. Conclusión: La suplementación siguiendo las recomendaciones de la ACOG y la SEGO es efectiva en el control de colesterol LDL, HDL, folato sérico, vitamina B12, homocisteína, hemoglobina, creatinina y plaquetas. Sólo los niveles de triglicéridos no se relacionan con la suplementación y pueden estar relacionados con mayor incidencia de colestasis y preeclampsia en gestaciones múltiples.

\section{PALABRAS CLAVE: Gestación gemelar, suplementación, triglicéridos, metabolismo}

\section{SUMMARY}

Background: Different ways of nutritional supplementation are being advised by physicians during pregnancy, but there is a lack of literature focused on its metabolical results. Aims: Check if the control of the impact that the multiple metabolic changes related to multiple pregnancies have on the mother's homeostasis is achieved with the supplementation, through common used blood tests. Method: We compare three different groups of patients between the 25 and 27th week of gestation. One group of 46 single pregnancies, a second group of 38 spontaneous twin pregnancies and another group of 32 twin pregnancies after assisted reproduction techniques. We compare the levels of LDL, HDL-cholesterol, triglycerides, serum folic acid, Vit B12, homocysteine, haemoglobine, creatinine and platelets. Results: The levels of triglycerides were higher in both groups of twin pregnancies $(p<0.001)$, but the levels of cholesterol remain similar. Folic acid concentrations were higher in the groups of twin pregnancies $(p<0.001)$. The rest of levels studied were similar in 
the 3 groups. Conclusion: The supplementation following the ACOG and SEGO recommendations seems to be effective in controlling the values of LDL, HDL-cholesterol, serum folic acid, Vit B12, homocysteine, haemoglobine, creatinine and platelets. Only the levels of triglycerides appear unaffected by the supplementation and can be related with the higher incidence of preeclampsia and cholestasis seemed among twin pregnancies.

KEY WORDS: Twin pregnancy, supplementation, triglycerides, metabolism

\section{INTRODUCCIÓN}

La incidencia de embarazos múltiples ha aumentado a lo largo de las últimas dos décadas; este incremento se ha debido principalmente a las técnicas de reproducción asistida. Junto a este cambio ha sucedido un incremento en ciertas patologías, requiriéndose con ello nuevos estudios al respecto. Desde el punto de vista de las gestantes, los embarazos múltiples se relacionan con mayores tasas de anemia, diabetes gestacional, hiperémesis gravídi$\mathrm{ca}$, infecciones del tracto urinario, colestasis intrahepática, preeclampsia, síndrome de HELLP, hemorragia posparto, amenaza de parto prematuro, abruptio de placenta y endometritis puerperal (1).

Los cambios metabólicos que suceden a lo largo de una gestación han sido bien estudiados; son la suma de pequeños y continuos ajustes fisiológicos que afectan al metabolismo de todos los nutrientes, con el fin de proveer al feto para su correcto desarrollo, preparar a la madre para la lactancia y a su vez mantener la homeostasis materna (2). Los embarazos múltiples suponen exigencias mayores, por lo que se han diseñado diversas estrategias con el fin de guiar a estas gestantes hacia el mejor desarrollo y desenlace de su embarazo. Las pacientes son suplementadas con complejos multivitamínicos, de cara a afrontar las mayores necesidades metabólicas a las que se ven sometidas por la placenta y el desarrollo del feto. Así, se suplementan con yodo, ácido fólico, hierro y a menudo un variado pool de vitaminas. Además se les instruye a realizar una actividad física moderada y una dieta equilibrada rica en frutas y verduras.

Nuestro objetivo es estudiar si realmente logramos controlar el impacto de los cambios metabólicos sobre la homeostasis materna en las gestaciones gemelares en comparación con las gestaciones únicas mediante la suplementación vitamínica, las recomendaciones dietéticas y los consejos respecto a la actividad física.

\section{PACIENTES Y MÉTODO}

Se seleccionaron tres grupos de pacientes entre 20 y 45 años sin antecedentes personales de interés: un primer grupo (grupo control) compuesto por 46 gestaciones únicas seguidas en nuestras consultas, un segundo grupo de 38 gestaciones gemelares espontáneas que iniciaron suplementación tras acudir por primera vez a consultas de tocología, y un tercer grupo de 32 pacientes que lograron su gestación gemelar por técnicas de reproducción asistida (FIV o ICSI) y que habían comenzado con suplementación al menos desde 3 meses antes de la gestación. Todas las pacientes fueron seguidas en una consulta especializada en gestaciones múltiples y recibieron educación acerca de nutrición y dietética durante el embarazo por una enfermera experimentada. Se les realizó análisis sanguíneo en ayunas de al menos 12 horas entre las semanas 25 y 27 de gestación, que incluyó las siguientes determinaciones: hemograma, niveles de coagulación, homocisteína plasmática, niveles séricos de folato, vitamina B 12, creatinina, colesterol total, colesterol-LDL, colesterol-HDL, triglicéridos y glucemia.

La suplementación que recibieron todas las pacientes cumplía como mínimo con las recomendaciones de la Sociedad Española de Ginecología y Obstetricia (SEGO) respecto a ácido fólico (mínimo de $0,4 \mathrm{mg} / \mathrm{día}$ ) , yodo (mínimo $200 \mu \mathrm{g} /$ día) y hierro (30 mg de hierro elemental/día a partir de semana 20). Las gestaciones gemelares además fueron suplementadas según las recomendaciones de el American College of Obstetrics and Gynecology (ACOG) con $1 \mathrm{mg}$ de ácido fólico/día y $60 \mathrm{mg}$ de hierro elemental/día $(1,3)$.

El análisis estadístico se realizó con el test de t de Student para muestras independientes, al ser las variables analizadas continuas y de distribución normal. Se consideró un valor $\mathrm{p}<0,05$ como diferencia estadística significativa. 


\section{RESULTADOS}

Se trata de tres grupos homogéneos respecto a la edad, de modo que no existieron diferencias estadísticamente significativas entre ellos en cuanto a la edad de las pacientes. Los niveles de folato sérico están significativamente elevados en las gestaciones gemelares bien hayan sido logradas espontáneamente o mediante técnicas de reproducción asistida respecto a las únicas $(p<0,001)$. Respecto a la vitamina B12, los valores más altos fueron los del grupo de gestaciones gemelares por técnicas de reproducción asistida, seguidos de las gestaciones únicas y los valores más bajos se registraron en las gestaciones gemelares espontáneas, aunque no existía diferencia significativa entre los grupos de gestaciones gemelares al compararlos con el grupo de gestaciones únicas. Los valores de homocisteína fueron mayores en ambos grupos de gestaciones gemelares respecto a las gestaciones únicas $(p<0,05)$. Los resultados se encontraron dentro del rango de valores normales en todos los grupos y pacientes (Tablas I y II).

Tabla I

ANÁLISIS COMPARATIVO ENTRE GESTACIÓN ÚNICA Y GESTACIÓN GEMELAR ESPONTÁNEA

\begin{tabular}{lccr}
\hline Variables & $\begin{array}{c}\text { Gestación única } \\
\text { Media (rango) } n=46\end{array}$ & $\begin{array}{c}\text { Gestación gemelar espontánea } \\
\text { Media (rango) } n=38\end{array}$ & Valor $p$ \\
\hline Edad (años) & $33,9(27-43)$ & $34,2(20-43)$ & $>0,05$ \\
Hemoglobina $(\mathrm{g} / \mathrm{dl})$ & $11,8(9,8-15)$ & $12,1(9,7-15,1)$ & $>0,05$ \\
Plaquetas $(10 \wedge 3 / \mathrm{ll})$ & $232,5(144-346)$ & $209,9(124-405)$ & $>0,05$ \\
Glucemia $(\mathrm{mg} / \mathrm{dl})$ & $82,6(64-100)$ & $81,8(68-111)$ & $>0,05$ \\
Creatinina $(\mathrm{mg} / \mathrm{dl})$ & $0,7(0,5-0,9)$ & $0,7(0,5-1)$ & $>0,05$ \\
Colesterol total $(\mathrm{mg} / \mathrm{dl})$ & $273,9(182-379)$ & $278,2(201-394)$ & $>0,05$ \\
Colesterol-LDL $(\mathrm{mg} / \mathrm{dl})$ & $166,6(80-253)$ & $171,9(123-271)$ & $>0,05$ \\
Colesterol-HDL $(\mathrm{mg} / \mathrm{dl})$ & $67,9(44-98)$ & $63,5(47-90)$ & $>0,05$ \\
Triglicéridos $(\mathrm{mg} / \mathrm{dl})$ & $170,4(105-373)$ & $236,3(130-474)$ & 0,0001 \\
Folato sérico $(\mathrm{ng} / \mathrm{ml})$ & $15,3(8,2->20)$ & $18,6(12,6->20)$ & $<0,001$ \\
Vit. B12 $(\mathrm{pg} / \mathrm{ml})$ & $277,9(166-555)$ & $263,6(134-613)$ & $>0,05$ \\
Homocisteína $(\mu \mathrm{mol} / \mathrm{l})$ & $3,9(2,3-7,1)$ & $4,3(2,1-8,2)$ & 0,034 \\
\hline
\end{tabular}

Tabla II

ANÁLISIS COMPARATIVO ENTRE GESTACIÓN ÚNICA Y GESTACIÓN GEMELAR POR REPRODUCCIÓN ASISTIDA

\begin{tabular}{lccc}
\hline Variables & $\begin{array}{c}\text { Gestación única } \\
\text { Media (rango) } \\
\mathrm{n}=46\end{array}$ & $\begin{array}{c}\text { Gestación gemelar } \\
\text { por reproducción asistida } \\
\text { Media (rango) } \\
\mathrm{n}=32\end{array}$ & $\begin{array}{c}\text { Valor } \mathrm{p} \\
\text { Edad (años) }\end{array}$ \\
Hemoglobina $(\mathrm{g} / \mathrm{dl})$ & $33,9(27-43)$ & $34,9(27-48)$ & $>0,05$ \\
Plaquetas $(10 \wedge 3 / \mathrm{ll})$ & $11,8(9,8-15)$ & $12,1(9,7-14,4)$ & $>0,05$ \\
Glucemia $(\mathrm{mg} / \mathrm{dl})$ & $232,5(144-346)$ & $210,3(130-329)$ & $>0,05$ \\
Creatinina $(\mathrm{mg} / \mathrm{dl})$ & $82,6(64-100)$ & $79,2(60-107)$ & $>0,05$ \\
Colesterol total $(\mathrm{mg} / \mathrm{dl})$ & $0,7(0,5-0,9)$ & $0,7(0,6-0,9)$ & $>0,05$ \\
Colesterol-LDL $(\mathrm{mg} / \mathrm{dl})$ & $273,9(182-379)$ & $277,5(150-498)$ & $>0,05$ \\
Colesterol-HDL $(\mathrm{mg} / \mathrm{dl})$ & $166,6(80-253)$ & $165,9(84-339)$ & $>0,05$ \\
Triglicéridos $(\mathrm{mg} / \mathrm{dl})$ & $67,9(44-98)$ & $63,4(42-90)$ & $>0,05$ \\
Folato sérico $(\mathrm{ng} / \mathrm{ml})$ & $170,4(105-373)$ & $249,5(153-407)$ & $<0,0001$ \\
Vit. B12 $(\mathrm{pg} / \mathrm{ml})$ & $15,3(8,2->20)$ & $19,1(15,1->20)$ & $<0,001$ \\
Homocisteína $(\mu \mathrm{mol} / \mathrm{l})$ & $277,9(166-555)$ & $306,4(176-689)$ & 0,057 \\
\hline
\end{tabular}


En cuanto al metabolismo lipídico existió una diferencia estadísticamente significativa en los niveles de triglicéridos. El nivel medio de triglicéridos se situó en 236,3 mg/dl en el grupo de gestaciones gemelares espontáneas, y fue mayor en el grupo de reproducción asistida (valor medio de $249,5 \mathrm{mg}$ / dl); mientras que el nivel medio fue de $170 \mathrm{mg} / \mathrm{dl}$ en las gestaciones únicas $(p<0,0001$ en la comparación con ambos grupos de gemelares). Los valores de colesterol total y colesterol-LDL se encontraron elevados, aunque sin diferencias significativas entre los grupos de gemelares y el de gestaciones únicas. Los niveles de colesterol-LDL se encontraron próximos a niveles de $160 \mathrm{mg} / \mathrm{dl}$, lo que consideramos límite superior de normalidad en mujeres no gestantes. El nivel de colesterol-HDL fue similar en los 3 grupos con valores medios dentro del rango de normalidad (Tablas I y II).

Los niveles de hemoglobina, plaquetas, creatinina y glucemia basal en ayunas fueron similares en los grupos de gemelares y en el de gestaciones únicas (Tablas I y II).

\section{DISCUSIÓN}

El estudio muestra como la suplementación con ácido fólico y vitamina B12 llevado a cabo con todas las pacientes, además de las medidas dietéticas, lograron valores adecuados de ácido fólico y vitamina B12 en todos los grupos; si bien ya sea por una suplementación mayor o por mayor adherencia al tratamiento los grupos de gemelares presentan valores más altos de ácido fólico en suero. La homocisteína aparece dentro de valores de normalidad en todos los grupos y pacientes, si bien su valor es mayor en el caso de gestaciones gemelares. La suplementación con ácido fólico y vitamina B12 explica que ningún paciente tuviera valores fuera de rangos de normalidad (4). Otros autores ya han intentado correlacionar los niveles de homocisteína entre gestaciones gemelares y únicas; han concluido también que los niveles de homocisteína no difieren si se ha establecido suplementación previa (5).

En cuanto al metabolismo lipídico, el hecho de que los niveles de colesterol- LDL se situasen próximos a la normalidad y sin diferencias entre grupos, y que los de colesterol-HDL se situarán dentro del rango normal, a pesar del incremento fisiológico que sucede de las diferentes lipoproteínas en el tercer trimestre de gestación, se puede explicar a través de la suplementación con ácido fólico, ya que está demostrado que los valores plasmáticos de folatos favorecen un mejor perfil lipoproteico disminuyendo los valores de colesterol-LDL y elevando los de colesterol-HDL (6).
En cuanto a los niveles de triglicéridos, la suplementación ya sea antes de la concepción o posconcepcional, no parece modificar la influencia que una gestación doble tiene sobre los niveles de triglicéridos (de hecho los niveles de triglicéridos fueron mayores entre las gestantes de gemelares que recibieron suplementación por más tiempo). Los triglicéridos en el tercer trimestre de gestación se elevan a causa de la mayor actividad de la lipasa hepática estimulada por los estrógenos, del aumento en la captación hepática de ácidos grasos libres provenientes de la lipólisis del tejido adiposo de reserva y de la disminución de la actividad lipoproteinlipasa del tejido adiposo (7). Además, la progesterona es responsable del aumento en el apetito a través de su influencia sobre el hipotálamo, siendo los niveles de progesterona mayores en gestaciones múltiples y a su vez por tanto mayores sus efectos a nivel hipotalámico (8). Este aumento de triglicéridos podría jugar un papel en el mayor riesgo de preeclampsia existente en gestaciones gemelares; esta influencia se explicaría por el desbalance entre sustancias oxidantes y antioxidantes que se produce en casos de hipertrigliceridemia mediante el mecanismo de peroxidación lipídica (9). Sin embargo, si bien evidenciamos niveles mayores de triglicéridos en las gestaciones gemelares, estos no fueron mayores a las elevaciones del 200 a $400 \%$ que llegan a considerarse fisiológicas a lo largo del embarazo (10). Además una elevación mayor de triglicéridos en gestaciones gemelares podría correlacionarse fisiológicamente con una mayor necesidad futura de producción de leche materna.

Los valores similares de hemoglobina en los 3 grupos se pueden justificar por el seguimiento al que se someten estas pacientes suplementándose además con hierro según recomendaciones de la SEGO y del ACOG. Por otro lado, los niveles similares de creatinina y plaquetas entre los 3 grupos resultan esperables, ya que no existe evidencia de que gestaciones gemelares deban repercutir en estos valores en el inicio del tercer trimestre, si bien la incidencia de síndromes que pueden afectar estos valores como el de HELLP son más prevalentes en gestaciones múltiples (11). Los valores de la glucemia basal sin diferencias significativas entre grupos y dentro del rango de valores normales, ponen de manifiesto que las pacientes no presentaban alteraciones marcadas en el metabolismo de carbohidratos en el momento del estudio, si bien el análisis se realizaba antes que el test de O'Sullivan y no se tuvo en cuenta la incidencia de diabetes gestacional en los distintos grupos. 


\section{CONCLUSIÓN}

Este estudio pone de manifiesto el éxito de un buen control metabólico, de la suplementación y de la labor de una consulta especializada en gestaciones gemelares. Los niveles de las pruebas que se realizan de forma usual para el control metabólico durante la gestación son similares a los que se obtienen en gestaciones simples. Los triglicéridos no se alteran mediante la suplementación y pueden estar relacionados con consecuencias adversas para la paciente.

\section{BIBLIOGRAFÍA}

1. American College of Obstetricians and Gynecologists. Multiple gestation: complicated twin, triplet, and highorder multifetal pregnancy. Practice Bulletin 56. Washington DC. ACOG, 2004.

2. King JC. Physiology of pregnant and nutrient metabolism. Am J Clin Nutr 2000;71:1249S-55S.

3. Sociedad Española de Ginecología y Obstetricia (SEGO). Protocolo: Control prenatal del embarazo normal. Julio 2010. www.sego.es

4. Katre P, Bhat D, Lubree H, Otiv S, Joshi S, Joglekar C, Rush E, Yajnik C. Vitamin B12 and folic acid supplementation and plasma total homocysteine concentrations in pregnant Indian women with low B12 and high folate status. Asia Pac J Clin Nutr 2010;19:335-43.

5. Powers RW, Dunbar MS, Laivuori HM, Harger GF, Lykins DL, Roberts JM. Maternal plasma homocysteine concentrations are not increased in twin pregnancies. Hypertens Pregnancy 2005;24:49-58.

6. Semmler A, Moskau S, Grigull A, Farmand S, Klockgether T, Smulders Y, Blom H, Zur B, Stoffel-Wagner $B$, Linnebank M. Plasma folate levels are associated with the lipoprotein profile: a retrospective database analysis. Nutr J 2010;28;9:31.

7. Osorio O. Metabolismo de los lípidos durante el embarazo. Rev Colomb Obstet Ginecol 2000;51:113-7.

8. Gür S, Türk G, Demirci E, Yüce A, Sönmez M, Ozer $S$, Aksu EH. Effect of Pregnancy and foetal number on diameter of corpus luteum, maternal progesterone concentration and oxidant/antioxidant balance in ewes. Reprod Domest Anim 2011;46:289-95.

9. Martínez MP, Lozano JG. Hipertrigliceridemia y preeclampsia: papel fisiopatológico y evidencia actual. MedUNAB 2005;8:118-23.

10. Sattar N, Greer IA, Louden J, Lindsay G, McConnell M, Shepherd J, Packard CJ. Lipoprotein subfraction changes in normal pregnancy: threshold effect of plasma triglyceride on appearance of small, dense low density lipoprotein. J Clin Endocrinol Metab 1997;82:2483-91.

11. Hardardottir H, Kelly K, Bork MD, Cusick W, Campbell WA, Rodis JF. Atypical presentation of preeclampsia in high-order multifetal gestations. Obstet Gynecol 1996;87:370-4. 